\title{
The multi-spectral imaging diagnostic
}

Cite as: Rev. Sci. Instrum. 89, 103503 (2018); https://doi.org/10.1063/1.5058224

Submitted: 18 September 2018 . Accepted: 19 September 2018 . Published Online: 11 October 2018

B. L. Linehan, R. T. Mumgaard, M. Wensing, K. Verhaegh (D), Y. Andrebe, J. R. Harrison, B. P. Duval, C. Theiler (D), and TCV Team

\section{ARTICLES YOU MAY BE INTERESTED IN}

Filamentary velocity scaling validation in the TCV tokamak

Physics of Plasmas 25, 072506 (2018); https://doi.org/10.1063/1.5038019

MIRRORS: A MATLAB ${ }^{\circledR}$ GUI for temperature measurement by multispectral imaging radiometry

Review of Scientific Instruments 89, 104903 (2018); https://doi.org/10.1063/1.5041360

Cavity ring-down spectroscopy to measure negative ion density in a helicon plasma source for fusion neutral beams

Review of Scientific Instruments 89, 103504 (2018); https://doi.org/10.1063/1.5044504

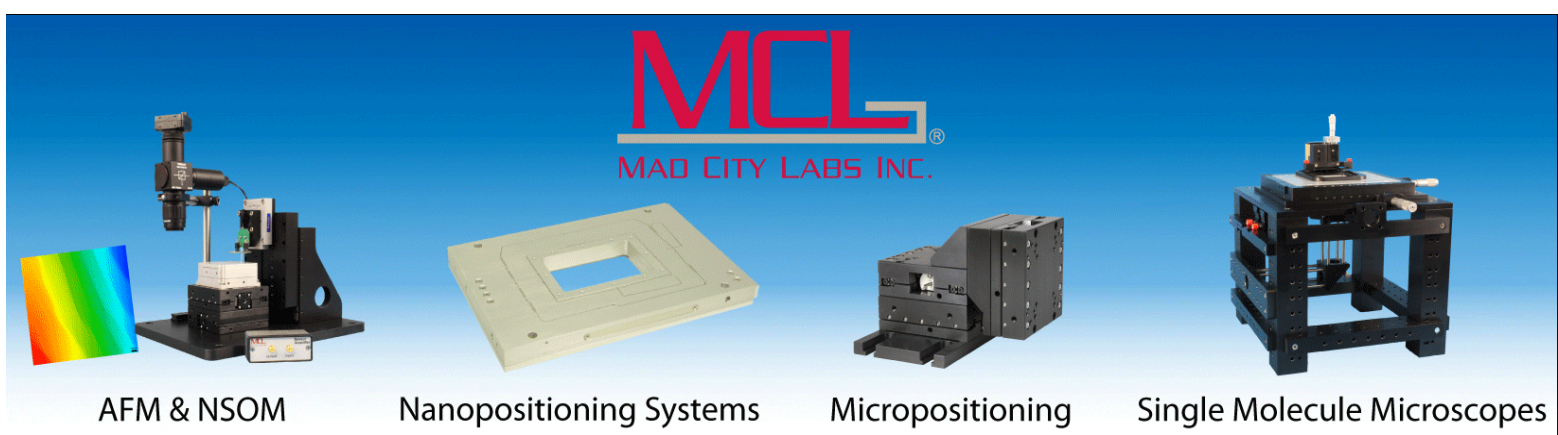




\title{
The multi-spectral imaging diagnostic
}

\author{
B. L. Linehan, ${ }^{1, a)}$ R. T. Mumgaard, ${ }^{1}$ M. Wensing, ${ }^{2}$ K. Verhaegh, ${ }^{2,3}$ Y. Andrebe, ${ }^{2}$ \\ J. R. Harrison, ${ }^{4}$ B. P. Duval, ${ }^{2}$ C. Theiler, ${ }^{2}$ and TCV Team ${ }^{\text {b) }}$ \\ ${ }^{1}$ Plasma Science and Fusion Center MIT, Cambridge, Massachusetts 02139, USA \\ ${ }^{2}$ École Polytechnique Fédérale de Lausanne (EPFL), Swiss Plasma Center (SPC), \\ CH-1015 Lausanne, Switzerland \\ ${ }^{3}$ York Plasma Institute, University of York, York, United Kingdom \\ ${ }^{4}$ Culham Centre for Fusion Energy, Culham Science Centre, Culham OX14 3DB, United Kingdom
}

(Received 18 September 2018; accepted 19 September 2018; published online 11 October 2018)

\begin{abstract}
The Multi-Spectral Imaging system is a new diagnostic that captures simultaneous spectrally filtered images from a common line of sight while maintaining a large étendue and high throughput. Imaging several atomic line intensities simultaneously may enable numerous measurement techniques. By making a novel modification of a polychromator layout, the MSI sequentially filters and focuses images onto commercial CMOS cameras while exhibiting minimal vignetting and aberrations. A four-wavelength system was initially installed and tested on Alcator C-Mod and subsequently moved to TCV. The images are absolutely calibrated and spatially registered enabling 2D mappings of atomic line ratios and absolute line intensities. The spectral transmission of the optical system was calibrated using an integrating sphere of known radiance. The images are inverted by crossreferencing points on TCV with a computer-aided design (CAD) model. Published by AIP Publishing. https://doi.org/10.1063/1.5058224
\end{abstract}

\section{INTRODUCTION}

Measurement of atomic emission is fundamental to the understanding of plasma and plasma surface interaction behavior. However, spectrometers are limited to one-dimensional measurements along a line of sight and can only observe one spectral region at a time. When spectral observations over 2D space are needed, it is a common practice to view the plasma with a camera through an interference filter. ${ }^{1,2}$ Clearly, this approach does not retain the high spectral resolution of a spectrometer or the ability to observe multiple spectral regions. Previously, multiple spectral regions have been imaged simultaneously by using beam splitters or dichromatic mirrors in combination with filters and cameras. ${ }^{3-5}$ However, since beam splitters and dichromatic mirrors bifurcate light, it is difficult to image many spectral regions simultaneously while maintaining high throughput.

The Multi-Spectral Imaging (MSI) diagnostic is a new diagnostic that uses a novel polychromator layout with interference filters and CMOS industrial cameras to create four simultaneous spectrally filtered images in the $390 \mathrm{~nm}-730 \mathrm{~nm}$ range. The system can distinguish light simultaneously anywhere in this range through interference filters with FWHM's less than $1 \mathrm{~nm}$. The polychromator approach uses light more efficiently than those employing dichromatic mirrors and beam splitters since interference filters pass light through a narrow bandpass and reflect the light outside of it. Therefore, the polychromator approach used by the MSI can be extended to many more wavelengths than has previously been practical with beam splitters and dichromatic mirrors. While the four wavelength systems described in this paper may not be

a)blinehan@mit.edu

b)See author's list of S. Coda et al., Nucl. Fusion 57, 102011 (2017). beyond the scope of a system employing dichromatic mirrors or beam splitters, future polychromator based diagnostics will surpass their scope. For example, a sixteen wavelength system has already been designed for NSTX-U by MIT, and another ten wavelength system has been installed at TCV by the Dutch Institute for Fundamental Energy Research.

\section{POLYCHROMATOR DESIGN}

The MSI polychromator is a modification of the polychromator design developed for the Motional-Stark Effect (MSE) diagnostic on Alcator C-MOD. ${ }^{6}$ It is designed to operate in the $390 \mathrm{~nm}-730 \mathrm{~nm}$ regime at an étendue of $9 \mathrm{~mm}^{2} \mathrm{sr}^{7}$ In modifying the MSE polychromator for the MSI, the relay lenses have been replaced with achromatic doublets which alleviate chromatic aberrations, the condensing lenses have been replaced with 5 element commercial camera lenses operating at $f / \# \geq 2.8$, and the avalanche photo-diode detectors have been replaced with low-cost commercial CMOS cameras. See Table I for a description of the cameras, and see Fig. 1 for a ray tracing of the system.

TABLE I. Description of camera features.

\begin{tabular}{lc}
\hline \hline $\begin{array}{l}\text { Camera model } \\
\text { Property }\end{array}$ & $\begin{array}{c}\text { Basler Ace acA1920-155 } \mu \mathrm{m} \\
\text { Value }\end{array}$ \\
\hline Sensor dimensions & $11.3 \times 7.1 \mathrm{~mm}$ \\
\# Pixels & $1920 \times 1200$ \\
Pixel size & $5.86 \mu \mathrm{m} \times 5.86 \mu \mathrm{m}$ \\
Minimum exposure & $20 \mu \mathrm{s}$ \\
Gain & $0-36 \mathrm{~dB}$ \\
Max framerate at 8 bits & $165 \mathrm{fps}$ \\
Max framerate at 12 bits & $88 \mathrm{fps}$ \\
\hline \hline
\end{tabular}




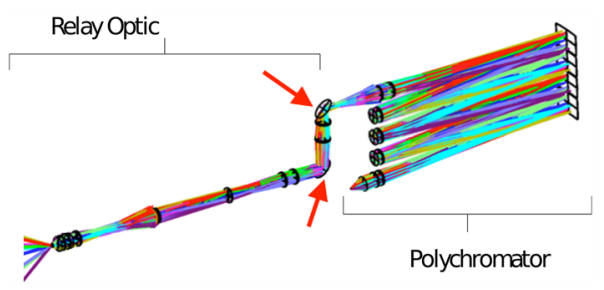

FIG. 1. Ray tracing of the MSI system. The arrows highlight the adjustable mirrors in the relay optic that can be used to adjust the view.

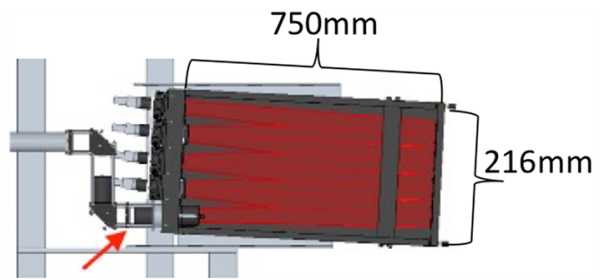

(a)

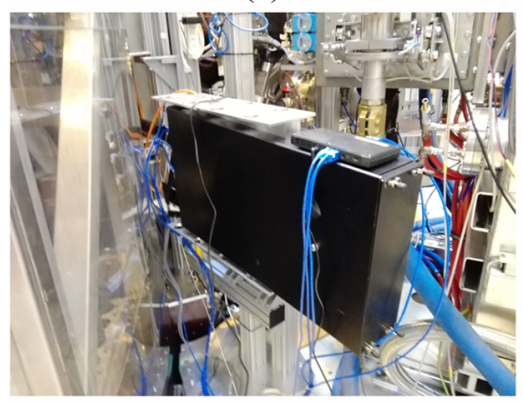

(b)

FIG. 2. (a) Drawing of the combined the MSI polychromator and relay optic system. The arrow indicates the placement of the intermediate removable target which is used for alignment. (b) Picture of the MSI polychromator and relay optic installed at TCV.

The performance of the MSI system is dependent on the quality of the filters. It is important that a filter be narrow enough to isolate the atomic line of interest, but wide enough so that the relative transmission of the filter does not change appreciably over the width of the atomic line. The spectrum transmitted through the interference filters is sensitive to the average angle of incidence from the normal angle, $\theta$, and to the cone angle, $\alpha$, of the light. Both $\theta$ and $\alpha$ will cause the center wavelength of an interference filter to be shifted to the blue and the shape of the transmitted spectrum to be widened. ${ }^{6}$
The filters in the polychromator filter light at an average angle of incidence of about $3^{\circ}$ from normal. To minimize these shifts, filters purchased specifically for the MSI have their centerwavelength specified for light incident at $3^{\circ}$ from normal with a FWHM of about $1 \mathrm{~nm}$.

\section{INSTALLATION AND RELAY OPTIC}

The MSI was installed on TCV in July of 2017. It views the divertor region of the tokamak on a tangential line of sight. The field of view covers both divertor legs and the X-point.

An imaging relay optic was made to interface the MSI polychromator to the TCV tokamak. See Fig. 2 for a drawing and photo of the installation. The key features of the relay optic are as follows:

- It creates a real image immediately before the entrance to the polychromator. This allows a transparent and removable target to be place immediately before the aperture which facilitates the alignment of the cameras to one another.

- The relay optic is designed like a periscope containing two adjustable mirrors which steer the relay optic output into the polychromator entrance (see Fig. 1).

\section{SYSTEM PERFORMANCE}

A Zemax ray tracing for the relay optic and polychromator was performed to investigate the performance of the system. A sketch of the rays going through the system is shown in Fig. 1, and the results of the Geometrical Optical Transfer Function at the last camera are plotted in Fig. 3. The plot shows that the tangential ray has a resolution of $\sim 10 \mu \mathrm{m}$ ( 2 pixels) and that the sagittal ray has a resolution of $\sim 30 \mu \mathrm{m}$ ( 6 pixels). The magnification at $1 \mathrm{~m}$ from the front of the relay optics, which corresponds to the tangent point in the plasma, is 239. Note that the filters have been assumed to be perfect reflectors and the condensing lens has been assumed to be wide enough to accept all the rays.

In Fig. 4, an image taken during operation at TCV is shown. The four simultaneous images display the $\mathrm{D}_{7-2}$ (397 nm), D $\mathrm{D}_{5-2}(434 \mathrm{~nm}), \mathrm{NII} 2 \mathrm{~s}^{2} 2 \mathrm{p} 3 \mathrm{p} \rightarrow 2 \mathrm{~s}^{2} 2 \mathrm{p} 3 \mathrm{~s}(460 \mathrm{~nm})$, and CIII $1 s^{2} 2 s 3 p 3 p \rightarrow 1 s^{2} 2 s 3 s(465 \mathrm{~nm})$ atomic lines. The shape of the lower single null plasma is clear in all four images. Features such as the intersection of tiles in the tokamak can

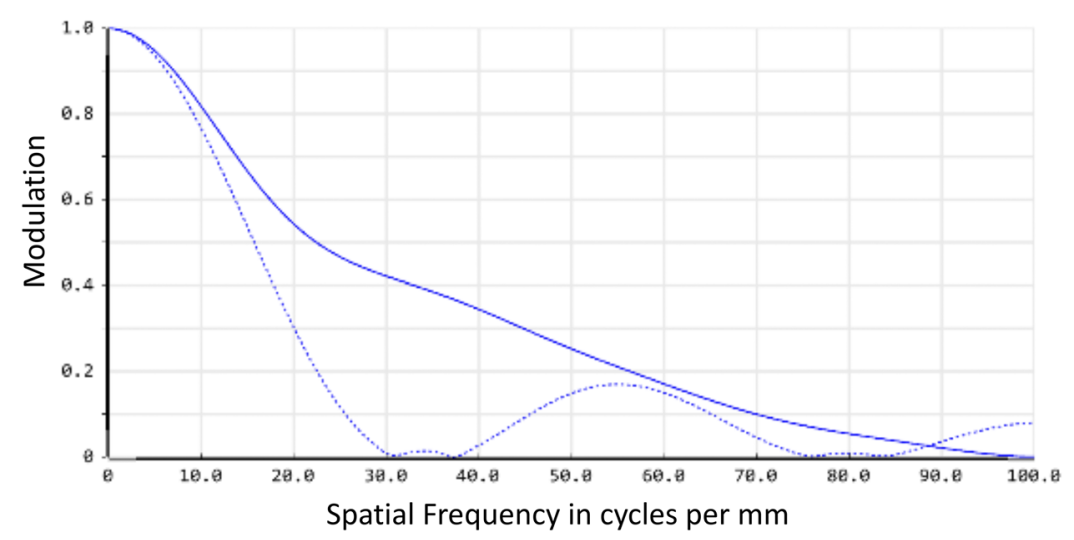

FIG. 3. The geometrical modular transfer function of the MSI system (polychromator and relay optic) at the center of the last camera as calculated by Zemax. The geometrical approximation is justified since the optical system is far from the diffraction limit. The tangential ray intercepts the $\mathrm{x}$-axis at $\sim 100$ lines/mm giving a resolution of $\sim 10 \mu \mathrm{m}$ ( 2 pixels), and the sagittal ray intercepts the $\mathrm{x}$-axis at $\sim 30$ lines/mm giving a resolution of $\sim 33 \mu \mathrm{m}$ (6 pixels). 


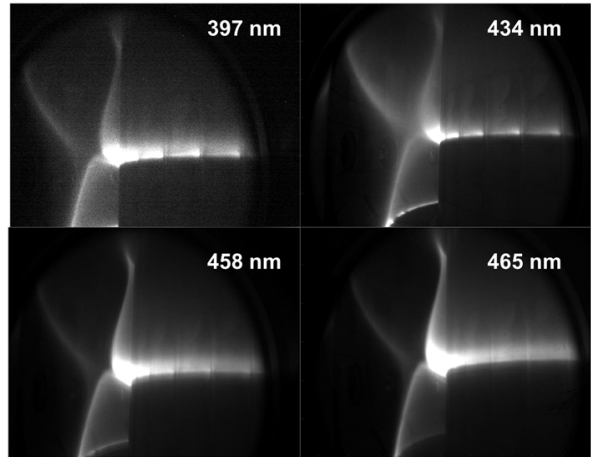

FIG. 4. Image taken by the MSI at TCV in September 2017. The images have slightly different field of views due to the fact that the detectors are in different positions with regards to their respective channels in the polychromator.

be distinguished and compared to their known position in a CAD model. This comparison allows for the inversion of the images. ${ }^{8}$

\section{CALIBRATION AND SPATIAL REGISTRATION}

The MSI is calibrated using an incandescent lamp directed through an integrating sphere and then into the MSI. The spectral radiance of the integrating sphere is known, so the transmission efficiency for each pixel through a given filter can be calculated. This calibration allows for a measurement of absolute radiance of the plasma over a spectral regime and a measurement of relative radiance between spectral regimes.

Three integrating spheres were used to calibrate the MSI. Their uncertainties were specified by the manufacturer, who calibrated the spheres to $\pm 10 \%$. The calibration factors produced from the 3 different integrating spheres agreed to within $5 \%$. From this, it is inferred that line ratio measurements made with the MSI are accurate to about $\pm 5 \%$ and the absolute measurements are accurate to within $\pm 10 \%$. Work is ongoing to refine the calibration to decrease uncertainties. The system was calibrated with different configurations of the relay optics to look for sensitivities and these were found at the $\pm 10 \%$ level. Finally, because a calibration factor is determined for a single pixel, it is paramount that the optical system stays rigid when moving from bench calibration to installation. Ideally, the MSI would be calibrated after installation with an integrating sphere shined in from the vessel. This however is not always practical and has not yet been performed for this system.

The spectral transmission of the narrow-bandpass filters in operation was calibrated with a high resolution spectrometer. ${ }^{9}$ An optical fiber leading to the spectrometer was used in place of a camera, while light from an incandescent lamp was directed through an integrating sphere and then into the MSI. Data were recorded both with and without the interference filters in front of the camera to measure the shape of the spectrum transmitted by each filter. The blue shifts of the center wavelengths of the filters when operated in the polychromator were between $1 \mathrm{~nm}$ and $0.7 \mathrm{~nm}$, and the broadening of the FWHM's of the filters were negligible when operating in the polychromator. The narrowest filter currently implemented by the MSI was observed to have a FWHM of $0.87 \mathrm{~nm}$ at a center

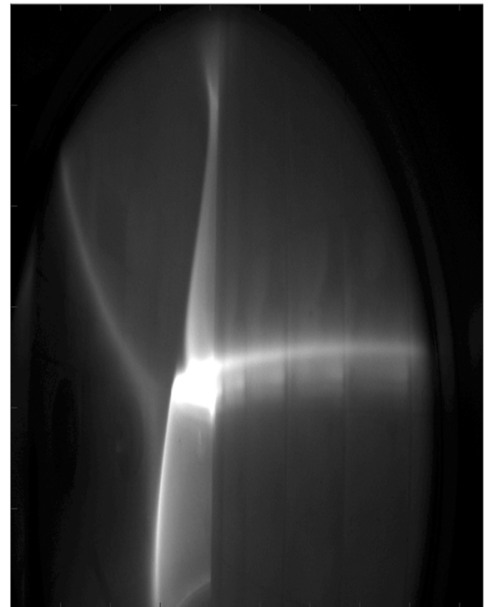

(a)
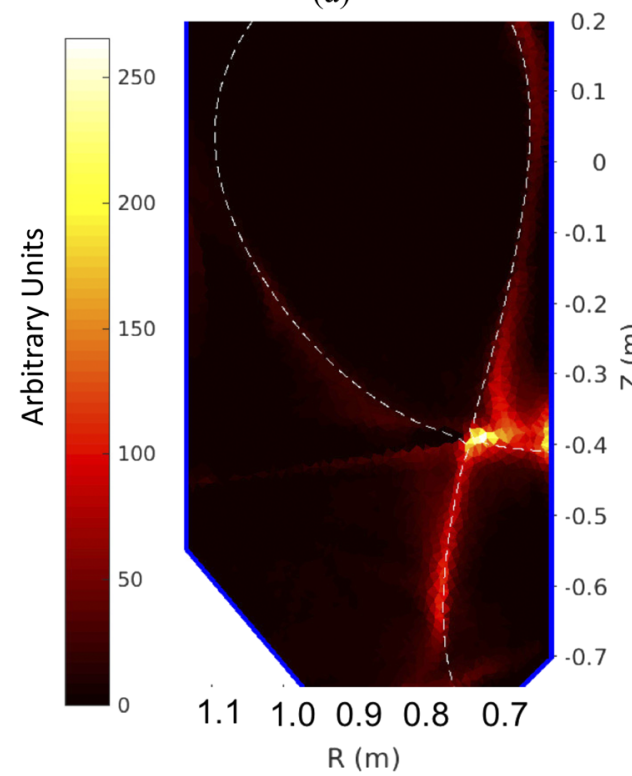

(b)

FIG. 5. (a) Raw image taken by the MSI camera. (b) Reconstruction of the raw image using SART. The dotted line traces the last closed flux surface. The line cutting horizontally across the X-point is clearly an artifact of the inversion. The absolute radiance calibration has not been applied. The inversion assumes toroidal symmetry.

wavelength of $433.57 \mathrm{~nm}\left(\mathrm{D}_{5-2}\right)$ when used in the polychromator. The insight taken from this calibration was that the quality of the filters was not significantly degraded when implemented in the MSI.

To perform the spatial inversion, the real space positions of features in an image are taken from a CAD drawing of the TCV tokamak and used as inputs into the Simultaneous Algebraic Reconstruction Technique (SART) algorithm which creates the inversion assuming toroidal symmetry. ${ }^{8,10}$ An example of an image and its inversion is shown in Fig. 5. Artifacts are visible in the reconstruction. Going forward, work will continue to be performed in improving the inversion process.

\section{FILTER SPECIFICATIONS}

While all the MSI filters were purchased from the same manufacture with the company's standard specifications for 
$\sim 1 \mathrm{~nm}$ FWHM filters, the absorption of out-of-band light varied significantly amongst the filters. This absorption is wavelength dependent and can remove up to $45 \%$ of the light at nearby wavelengths. This loss of light is not prohibitive for a 4 wavelength system but will be an issue for systems with many more wavelengths. Filters with high out-of-band reflection $(>90 \%)$ are required.

\section{CONCLUSION OF FUTURE APPLICATIONS}

The multi-spectral imaging system is a new diagnostic installed at TCV that takes four simultaneous spectrally filtered images. The polychromator layout first implemented here has the potential to be extended to imaging several more $(\geq 10)$ wavelengths simultaneously. This will facilitate many new diagnostic techniques incorporating observations of relative and absolute atomic emission intensities. For example, recombination rates can be inferred from Balmer line intensities, ${ }^{9,11-13} T_{e}$ and $n_{e}$ can be calculated from the ratios of helium singlet and triplet lines, ${ }^{14-17}$ and detachment can be observed through the spatial variation of the intensity of the CIII $465 \mathrm{~nm}$ line along the divertor leg. 5,18

\section{ACKNOWLEDGMENTS}

This work was supported by U.S. DoE Award Nos. DEFC02-99ER54512 and DE-AC05-06OR23100 and the Swiss National Science Foundation. This work has been carried out within the framework of thesis, Massachusetts Institute of Technology, and has received funding from the Euratom research and training programme 2014-2018 under Grant Agreement No. 633053. The views and opinions expressed herein do not necessarily reflect those of the European Commission.
${ }^{1}$ M. Fenstermacher, S. Allen, N. Brooks, D. Buchenauer, T. Carlstrom, J. Cuthbertson, E. Doyle, T. Evans, P.-M. Garbet, R. Harvey et al., Phys. Plasmas 4, 1761 (1997).

${ }^{2}$ M. Groth, R. Ellis, N. Brooks, M. Fenstermacher, C. Lasnier, W. Meyer, and J. Moeller, Rev. Sci. Instrum. 80, 033505 (2009).

${ }^{3}$ A. Huber, S. Brezinsek, P. Mertens, B. Schweer, G. Sergienko, A. Terra, G. Arnoux, N. Balshaw, M. Clever, T. Edlingdon et al., Rev. Sci. Instrum. 83, 10D511 (2012).

${ }^{4}$ A. Leonard, T. Petrie, W. West, M. Fenstermacher, S. Allen, D. Hill, R. Isler, C. Lasnier, G. Porter, D. Whyte et al., J. Nucl. Mater. 266, 348 (1999).

${ }^{5}$ J. Harrison, W. Vijvers, C. Theiler, B. Duval, S. Elmore, B. Labit, B. Lipschultz, S. Van Limpt, S. Lisgo, C. Tsui et al., Nucl. Mater. Energy 12, 1071 (2017).

${ }^{6}$ R. Mumgaard, S. Scott, and M. Khoury, Rev. Sci. Instrum. 87, 11 E527 (2016).

${ }^{7}$ R. T. Mumgaard, Ph.D. thesis, Massachusetts Institute of Technology, 2015.

${ }^{8}$ S. Silburn, J. Harrison, M. Smithies, A. Wynn, T. Farley, and J. Cavalier, Calcam, https://euratom-software.github.io/calcam, 2018.

${ }^{9}$ K. Verhaegh, B. Lipschultz, B. Duval, J. Harrison, H. Reimerdes, C. Theiler, B. Labit, R. Maurizio, C. Marini, F. Nespoli et al., Nucl. Mater. Energy 12, 1112 (2017).

${ }^{10}$ A. H. Andersen and A. C. Kak, Ultrason. Imaging 6, 81 (1984).

${ }^{11}$ D. Lumma, J. Terry, and B. Lipschultz, Phys. Plasmas 4, 2555 (1997).

${ }^{12}$ J. Terry, B. Lipschultz, A. Y. Pigarov, S. Krasheninnikov, B. LaBombard, D. Lumma, H. Ohkawa, D. Pappas, and M. Umansky, Phys. Plasmas 5, 1759 (1998).

${ }^{13}$ R. Isler, N. Brooks, W. West, A. Leonard, G. McKee, and G. Porter, Phys. Plasmas 6, 541 (1999).

${ }^{14}$ M. Griener, J. M. Burgos, M. Cavedon, G. Birkenmeier, R. Dux, B. Kurzan, O. Schmitz, B. Sieglin, U. Stroth, E. Viezzer et al., Plasma Phys. Controlled Fusion 60, 025008 (2017).

${ }^{15}$ S. Lisgo, P. Börner, G. Counsell, J. Dowling, A. Kirk, R. Scannell, M. OMullane, D. Reiter, M. Team et al., J. Nucl. Mater. 390, 1078 (2009).

${ }^{16}$ S. Sasaki, S. Takamura, S. Watanabe, S. Masuzaki, T. Kato, and K. Kadota, Rev. Sci. Instrum. 67, 3521 (1996).

${ }^{17}$ O. Schmitz, I. Beigman, L. Vainshtein, B. Schweer, M. Kantor, A. Pospieszczyk, Y. Xu, M. Krychowiak, M. Lehnen, U. Samm et al., Plasma Phys. Controlled Fusion 50, 115004 (2008).

${ }^{18}$ B. Lipschultz, J. Goetz, B. LaBombard, G. McCracken, J. Terry, M. Graf, R. Granetz, D. Jablonski, C. Kurz, A. Niemczewski et al., J. Nucl. Mater. 220, 50 (1995). 